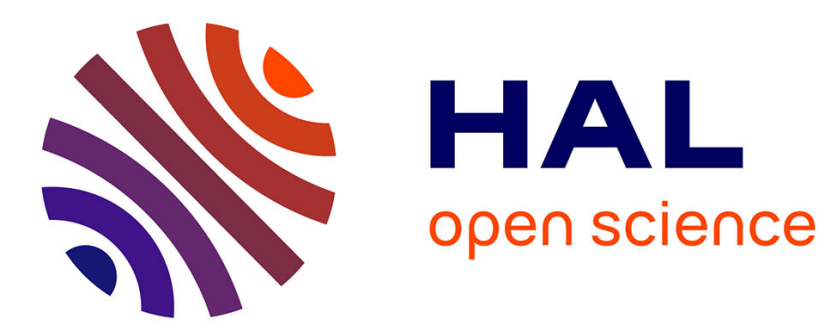

\title{
Comment évoluent les relations entre patients déments et proches aidants
}

Emmanuel Monfort

\section{To cite this version:}

Emmanuel Monfort. Comment évoluent les relations entre patients déments et proches aidants. NPG:

Neurologie - Psychiatrie - Gériatrie, 2019, 19 (122), pp.227-232. hal-02097014

\section{HAL Id: hal-02097014 \\ https://hal.univ-grenoble-alpes.fr/hal-02097014}

Submitted on 25 Oct 2021

HAL is a multi-disciplinary open access archive for the deposit and dissemination of scientific research documents, whether they are published or not. The documents may come from teaching and research institutions in France or abroad, or from public or private research centers.
L'archive ouverte pluridisciplinaire HAL, est destinée au dépôt et à la diffusion de documents scientifiques de niveau recherche, publiés ou non, émanant des établissements d'enseignement et de recherche français ou étrangers, des laboratoires publics ou privés.

\section{다)(1) $(5$}

Distributed under a Creative Commons Attribution - NonCommercial| 4.0 International 


\section{Comment évoluent les relations entre patients déments et proches aidants}

How the relationship between dementia patients and caregivers evolves

Emmanuel Monfort, Univ. Grenoble Alpes, LIP/PC2S (EA 4145), F-38000 Grenoble, France

Emmanuel Monfort, Univ. Grenoble Alpes, LIP/PC2S (EA 4145)

Maître de Conférences des Universités en Psychologie, Habilité à Diriger des Recherches

Laboratoire Inter-universitaire de Psychologie (LIP)

Univ. Grenoble Alpes

UFR SHS, 1251 avenue Centrale

BP 47

38040 Grenoble Cedex 9

France

Téléphone: +33 (0)4 76825912

Fax: $+33(0) 476825665$

Courriel : emmanuel.monfort@univ-grenoble-alpes.fr 


\section{Résumé}

De nombreux aidants de patients déments se sentent emprisonnés du fait d'obligations, de craintes, de peurs, qui les contraignent à rester enfermés, à la fois physiquement au domicile et psychologiquement. Ces difficultés impliquent de mettre en œuvre des processus d'adaptation d'un point de vue fonctionnel, mais aussi affectif et relèvent pour une large part de la relation à la personne dépendante. La qualité de la relation aidant-aidé constitue donc un facteur critique, qui détermine la nature de l'aide et le bien-être des aidants eux-mêmes, mais aussi les conditions de l'élaboration du rôle d'aidant, ainsi que de son évolution. Cette évolution est marquée par la survenue des événements marquants qui jalonnent la démence, ce qui implique de concevoir progressivement une nouvelle façon de concevoir leur proximité relationnelle avec le proche malade.

Mots-clés : Aidants ; famille ; démence ; relations

\section{Summary}

Informal caregivers of dementia patients often feel trapped because of their commitment, their worries and their fears, compelling them to remain confined to the home. These constraints imply the implementation of adjustment processes, both functional and emotional. The adaptation to these challenges is strongly conditioned by the relationship between the caregiver and the dependent person. Thus, the quality of the caregiver-care receiver relationship is a crucial factor that determines the nature of assistance provided and the well-being of the caregivers themselves. It also determines the elaboration of the role of the caregiver, and the way it will evolve. This evolution is affected by the occurrence of key events as the dementia progresses, which means that caregivers need to progressively come up with new ways of living with the demented person. 
Keywords: Caregivers; family; dementia; relationships 
Entre 2007 et 2060, la France est amenée à voir le nombre de personnes de 75 ans ou plus s'accroître de 6,7 millions et celui des 85 ans de 4,1 millions, faisant de leur prise en charge un enjeu pour les années futures [1]. A cela s'ajoute le fait que ce vieillissement constant de la population s'accompagne d'un accroissement de la prévalence des pathologies neurodégénératives et de la charge portée par leurs proches aidants, en raison des troubles physiques, cognitifs et comportementaux qu'entraînent ces pathologies [2]. Mais, au-delà des contraintes fonctionnelles associées à leur évolution, la charge ressentie par les aidants est également déterminée par une perte du sentiment de cohérence et par la faiblesse de la relation affective [3], ainsi que par la perception de changements dans les relations entre aidant et aidé [4], soulignant en cela l'importance des liens qui unissent les patients déments à leurs proches. Alors que les difficultés des aidants sont habituellement appréhendées au travers de la question du fardeau subjectif, c'est-à-dire de cette charge ressentie par les proches, et de ses liens au fardeau objectif, c'est-à-dire des troubles neurocognitifs et comportementaux des patients déments, nous présenterons les connaissances actuelles issues de la recherche concernant l'évolution des relations qui unissent les aidants à leurs proches parents souffrant de démence, depuis la reconnaissance des premières difficultés jusqu'aux derniers temps de l'accompagnement. A une perspective trop souvent trop fonctionnaliste, nous souhaitions ici ajouter une perspective relationnelle, essentielle pour mieux comprendre ce qui se joue au sein de la dyade aidant-aidé.

\section{Evolution des relations aidants-aidés}

Les premières difficultés rapportées par les aidants concernent une inquiétude anxieuse et envahissante, une interrogation perplexe, qui préfigure ce que sera par la suite la désorganisation de la relation [5]. A travers l'analyse de témoignages de conjointes de 
personnes affectées d'une pathologie neurodégénérative publiés entre 1993 et 2012, Ostrowski et Mietkiewicz [6] ont montré que ces préoccupations, souvent associées à du déni, inaugurent effectivement ce qui sera un cheminement particulier. Puis, avec le développement du rôle d'aidant, qui s'effectue pas à pas, la relation de couple glisse vers de l'interdépendance. L'accompagnement mobilise ensuite totalement le conjoint, celui-ci se positionnant comme le garant de la qualité de vie du proche malade et un expert de la dépendance. Enfin, l'entrée en institution est associée à des sentiments ambivalents, qui alternent entre une grande lucidité vis-à-vis des difficultés rencontrées et une culpabilité très prégnante. Nous verrons que ces récits autobiographiques sont le reflet des évolutions habituelles des relations aidants-aidés, mais qui ont pu être largement précisées par les recherches menées au cours des dernières années.

L'évolution des troubles neurocognitifs implique une perte progressive des liens à la personne malade, qui constitue une source de détresse majeure, les changements personnels, familiaux et existentiels auxquels sont confrontés les aidants débutant dès avant le diagnostic de la pathologie démentielle [7]. Avec la préparation aux situations d'aide et la prévisibilité des besoins à satisfaire, la valeur donnée par les proches de patients déments à la relation aidant-aidé prédit l'importance des contraintes qu'ils perçoivent dans l'exercice de leur rôle d'aidant [8]. La qualité de cette relation aidant-aidé est alors un facteur critique, qui détermine largement la qualité de l'aide et le bien-être des aidants eux-mêmes [9]. Interrogés sur leur perception de la vie au côté d'un malade d'Alzheimer, des conjoints rapportent se sentir emprisonnés, du fait d'obligations, de craintes, de peurs, qui les contraignent à rester enfermés au domicile, à la fois physiquement et psychologiquement. Même si certaines dimensions positives de la relation peuvent rester intactes, notamment l'amour, la chaleur humaine, l'intimité et l'affection [10], des changements opèrent au sein du couple aidant-aidé, au travers d'une diminution de la qualité de la communication et de la réciprocité des échanges, 
de l'opportunité de partager des activités et, d'une manière générale, de la satisfaction de la relation [11]. Les conjoints aidants évoquant parfois passer «de l'union à la solitude » [12], la question des pertes concernant donc à la fois la perte du conjoint et la perte de la relation maritale. Cette dimension affective de la relation au proche a pu être étudiée en faisant appel à la notion d'empathie. Il s'agit en effet de l'un des symptômes, rapportés par les proches aidants chez les patients déments auxquels ils viennent en aide, qui est jugé responsable d'une réelle atteinte de la qualité des relations, sans pourtant être associé au fardeau ressenti [13]. Un haut niveau d'empathie chez les aidants eux-mêmes est associé à moins de stress, de fardeau et de dépression [14]. Les conjoints de patients déments les plus empathiques accepteraient notamment mieux les évolutions observées chez leurs proches et auraient aussi des relations de meilleure qualité [15]. Plus particulièrement, un haut niveau d'empathie cognitive, c'est-à-dire une bonne compréhension de ce que ressent le proche dément est lié à une moindre humeur dépressive, mais un haut niveau d'empathie affective, c'est-à-dire un fort ressenti de ce que l'aidé ressent, est lié à une plus forte anxiété [16].

Pour mieux comprendre l'évolution des relations entre aidants et aidés, des modèles temporels des expériences vécues par les patients déments et leurs aidants ont été proposés. Keady [17] a par exemple décrit un modèle de l'aide qui tient compte à la fois de l'expérience de la personne malade et de celle de son proche, organisé en quatre temporalités successives : « travailler seul », « travailler ensemble », « travailler à côté », puis « travailler séparément ». Cette dernière étape peut être comparée à ce qui a été dénommé « perte ambiguë » [18] et qui correspond à un changement de la personnalité de l'aidant, par lequel il reste certes présent physiquement, mais absent psychiquement. Une évolution linéaire de la relation au sein du couple aidant-aidé ne reflète cependant pas toutes les relations d'aide aux patients déments. A partir d'entretiens réalisés sur une période de cinq ans, Hellstrom et al. [19] ont élaboré un modèle non linéaire, dans lequel les expériences de couples avec un partenaire souffrant de 
démence sont comprises non pas comme des étapes jalonnant l'évolution de la relation, mais comme des stratégies relationnelles, destinées à créer un « contexte relationnel nourrissant ». Ces stratégies sont généralement utilisées de façon graduelle, parallèlement à l'évolution de la maladie. Il s'agit en premier lieu de l'établissement d'une relation de couple soutenante (faire des choses, partager, rechercher le meilleur, etc.), puis du maintien de l'implication de l'aidant (jouer une part active dans la relation, laisser faire par d'autres, prendre des risques, etc.) et enfin du fait de passer à autre chose (rester un «nous », devenir un « je » et prendre un nouveau départ). Que ce soit dans le modèle proposé par Keady [17], ou dans ce celui formalisé par Hellstrom et al. [19], la dimension fondamentale est donc la qualité de la relation, que l'on peut situer sur un continuum, depuis une réelle collaboration destinée à faire face à la maladie jusqu'à une union au sein de laquelle les interactions sont pauvres. Favoriser le sentiment de continuité au travers de l'aide qui est apportée aux conjoints semble alors essentiel pour répondre à leurs difficultés.

Contrairement à une conception de la prise en charge en établissement pour personnes âgées dépendantes, centrée sur la perte d'autonomie et la santé des seuls patients souffrant de démence, une attention toute particulière devrait aussi être portée aux aidants familiaux de patients déments résidant en institution, pour limiter leur isolement, leur stigmatisation, leur culpabilité, la qualité de leurs relations et de ce fait, le stress qu'ils peuvent véhiculer. L'institutionnalisation, lorsqu'elle survient, constitue en effet une étape critique de la relation aidant-aidé. Bien que les aidants continuent fréquemment à éprouver des sentiments de détresse, de culpabilité, de stigmatisation et de perte au sein d'un système de soins auquel ils doivent s'adapter [20], les difficultés existentielles apparaissent comme bien différentes avant, puis après une entrée en institution [21]. Avant l'institutionnalisation, elles sont caractérisées par un sentiment de honte et de culpabilité, d'isolement, ainsi que de devoir mettre ses propres besoins entre parenthèses, plus important chez les descendants que chez les conjoints et 
associé à des symptômes dépressifs [22]. L'entrée en institution peut ensuite permettre d'atténuer les symptômes dépressifs des aidants de malades Alzheimer et d'accroître leur sentiment de contrôle sur leur propre existence [23]. Avec celle-ci, l'aidant doit néanmoins abandonner son statut d'aidant principal au profit d'une prise en charge plus médicalisée. Le processus qui conduit au renoncement au rôle qui préexistait jusqu'alors et qui doit, en définitive, aboutir à confier la responsabilité de la délivrance de l'aide à un professionnel, débute par un moment décisif qui justifie ce changement, puis se poursuit par une phase d'adaptation [24]. Il se traduit par une modification significative du rôle d'aidant, qui devient alors un superviseur de l'aide à apporter, pour enfin aboutir au renoncement de ce rôle. Ce n'est que lorsqu'il se trouve dégagé de ses obligations de soins, que ses expériences personnelles intimes, ses émotions et ses liens familiaux peuvent prendre une plus grande importance, ce qui lui permet notamment de limiter la perte des relations sociales qui était liée aux contraintes de l'accompagnement [22].

\section{Quel rôle pour les aidants}

Selon la théorie interactionniste du rôle [25], un aidant ne devient aidant qu'à partir du moment où il agit comme tel. En retour, ce rôle est influencé par les interactions qui opèrent entre l'aidant et l'aidé. Le rôle d'aidant est donc la conséquence d'une interaction au sein de la dyade aidant-aidé, qui débute dès la reconnaissance des premières difficultés, ainsi que par la préparation au rôle qui sera pris. La construction de ce rôle pourrait ensuite déterminer à long terme le devenir du proche souffrant de démence et notamment le risque d'institutionnalisation [26]. Gaugler et al. [26] ont pu montrer que les aidants endossent leur rôle à la suite de certains événements, qui varient selon les aidants. Il s'agit parfois de la seule annonce du diagnostic, de l'identification des symptômes de la démence suivie de l'annonce 
du diagnostic, de l'identification des symptômes suivie de la mise en œuvre de l'aide, ou encore de la mise en œuvre de l'aide, avant même que le diagnostic n'ait été posé et les troubles identifiés. L'étude de journaux écrits par des aidants remet quelque peu en question la conception selon laquelle le rôle d'aidant pourrait n'être endossé qu'après le diagnostic de la maladie [6]. Bien au contraire, elle a montré qu'ils acquièrent la fonction d'aidant dès la phase prédiagnostique, même si les changements personnels et relationnels opèrent de façon très graduelle et sont consolidés à la suite des rendez-vous avec les professionnels de santé.

Le lien à la personne atteinte de troubles neurocognitifs, implique une mise en œuvre du rôle d'aidant qui peut être différentes selon la nature de la relation qui les unit et implique donc aussi des conséquences différentes sur le bien-être, ou la santé. Les conjoints apportent plus fréquemment leur aide, mais ressentent aussi plus de difficultés que les enfants, ou que les beaux-enfants, étant plus engagés, avec un attachement émotionnel plus important aux personnes atteintes d'une maladie d'Alzheimer [27], avec un bouleversement de l'identité sociale qui touche les deux époux, mais plus particulièrement la capacité du conjoint aidant à rétablir un sentiment d'intimité au sein du couple [28].

La majorité des aidants paraissent considérer que la relation de couple demeure fondamentale pour le maintien de l'identité de soi, y compris lorsque le malade est institutionnalisé [29]. Les conjoints aidants de patients déments ont pu rapporter que la pathologie modifie leur expérience de la relation de couple, au travers de quatre thématiques : 1) l'expérience des pertes et de la solitude, 2) le changement de rôle, 3) l'altération de la communication et 4) les besoins d'aide et les stratégies d'adaptation associées à ces besoins [30]. Ils sont ainsi confrontés à des sentiments contradictoires, notamment du fait de se sentir contraints d'accompagner leur proche jusqu'à la fin et de souffrir du poids que représente cette aide. Si l'aidant parvient à préserver l'identité de son partenaire malade, alors c'est aussi l'identité de couple qui se voit préservée [31]. Certaines différences apparaissent cependant 
selon les sexes. En effet, les femmes semblent consacrer plus de temps au maintien de la dimension relationnelle [32] et éprouvent plus de difficultés à rétablir un sentiment d'intimité au sein du couple [31]. Avec une perspective plus fonctionnelle, les hommes pourraient d'avantage trouver un le sens à la responsabilité qu'ils endossent [32]. Quatre thèmes majeurs peuvent ainsi caractériser la relation d'une conjointe, ou d'un conjoint, au malade d'Alzheimer avec lequel elle, ou il, partage sa vie [33] : 1) la distance au conjoint dépendant (soit en terme de proximité, soit d'éloignement), 2) la tension qui peut exister entre la réponse aux besoins du conjoint et à ses propres besoins, 3) la recherche de stratégies, de modes de contrôle émotionnels ou des pratiques d'aide et 4) la connaissance, ou au contraire l'absence de connaissance des situations à venir.

Les enfants sont peu différents des beaux-enfants, bien que ces derniers paraissent avoir une perception moins positive de la relation d'aide que les enfants [34]. En revanche, les enfants et beaux enfants sont plus à risque que les conjoints de ressentir des conflits de rôles liés aux charges familiales et professionnelles qu'ils portent parallèlement à l'aide qu'ils apportent à leurs parents âgés. Ils peuvent également ressentir des sentiments de culpabilité [24] d'obligation et de devoir plus fort vis-à-vis de leur parent [35], contrairement aux conjoints, dont les motivations sont constitutives de la relation de couple. Selon un processus d'inversion des rôles, les enfants de patients déments peuvent alors devenir des figures d'attachement protectrices pour leurs parents âgés dépendants [36].

\section{Quelles adaptations relationnelles chez les aidants}

Le stress vécu dans le cadre de la relation aidant-aidé implique la mise en œuvre de modes d'adaptation Comme cela a pu être observé dans nombre de situations, les stratégies adaptatives (ou coping) centrées sur le problème, sont notamment associées à un meilleur 
bien-être des aidants et à une meilleure satisfaction vis-à-vis de leur rôle d'aidant, contrairement aux stratégies centrées sur les émotions [37]. En revanche, les stratégies d'évitement, qui visent à réduire l'expression de désirs illusoires concernant la possibilité que la pathologie démentielle cesse [38], sont associées à un fardeau plus important. Ces stratégies ne prédisent pourtant pas nécessairement l'anxiété, la dépression et le stress des aidants, non plus que leur propre évaluation de la qualité de l'aide qu'ils apportent [39]. A ce type de fonctionnement adaptatif peut s'adjoindre une réelle quête de liberté, qui passe par le recours à de nouvelles stratégies pour élaborer un «espace vécu » avec le proche malade, dans lequel l'aidant se réserve par exemple un espace personnel indépendant du proche dépendant, espace de répit qui lui permet ensuite d'être plus disponible et attentif pour les moments positifs [40].

Le système d'attachement, par nature relationnel, est une ressource adaptative essentielle pour la sécurité psychologique, dont l'importance apparaît clairement chez les proches aidants de patients déments. Alors que chez les aidants, un attachement insécure est prédictif de difficultés d'adaptation psychologique et de fardeau chez les aidants [41], un attachement sécure, soit un lien affectif de confiance, paraît avoir des conséquences notables sur la santé psychique des aidants [42]. Il permettrait particulièrement de protéger de l'influence négative des difficultés du quotidien [43]. Si les enfants aidants présentent un profil d'attachement insécure, ils sont alors en difficulté pour apporter leur aide, en restant centrés sur leurs propres besoins [36]. L'attachement dont font preuve les malades d'Alzheimer peut aussi influer sur la perception des symptômes de la maladie par leurs proches [44]. Ainsi, les patients qui manifestent un attachement anxieux présentent des symptômes physiques et psychologiques plus graves, d'autant plus que l'attachement de leur proche aidant est également de type anxieux. La qualité de l'attachement devrait donc être envisagée non pas de façon univoque, mais conjointement chez le malade d'Alzheimer et chez son proche, selon une approche relationnelle aidant-aidé. 
Comme pour l'ensemble des processus qui permettent de caractériser la situation des aidants, l'attachement doit être appréhendé selon une perspective évolutive. Il s'agit donc de prendre en compte l'histoire de la relation d'attachement au proche parent dément. Celle-ci peut en effet affecter les pensées, les sentiments, ainsi que les comportements des aidants, tels que leur implication dans les aides qu'ils apportent, par des conflits relationnels, ou encore dans leurs attitudes critiques [45]. Des connaissances procédurales sur les scripts sécures, c'est-à-dire concernant la manière et le contexte dans lesquels se manifeste un attachement sécure, sont également apparues comme associées à des relations conflictuelles moins importantes, mais aussi à une moindre implication dans la relation d'aide, soit à une relative distanciation. Le développement d'une certaine compréhension et de souvenirs concernant ce qui constitue la proximité relationnelle pourrait donc permettre de prendre le recul nécessaire pour apporter une aide efficiente. Cette évolution de la relation, telle qu'elle peut être souhaitée, a été qualifiée de «symbiose compensatoire progressive » [46], processus correspondant à l'association de trois composantes : la prise de conscience d'un déséquilibre dans la relation intime, l'engagement dans la relation d'aide et la mise en œuvre de mécanismes compensatoires. Par ailleurs, au-delà de la reconnaissance par les aidants qu'il peut se maintenir, voire se développer une relation d'attachement, des préoccupations sur l'attachement, telles que la crainte de la disparition du parent, ou des inquiétudes au sujet de l'attachement à la personne souffrant de démence, prédisent aussi des difficultés psychologiques, sans pour autant que cela n'accroisse le fardeau [41].

\section{Conclusion}

L'accompagnement des personnes âgées souffrant de pathologies neurodégénératives est une histoire relationnelle, marquée par une perte progressive de la qualité des interactions, 
mais dans laquelle des liens, notamment affectifs, peuvent néanmoins être maintenus. Cette histoire a montré qu'elle débute dès l'apparition des premiers signes de la maladie, puis se poursuit, en étant marquée par des étapes cruciales (l'annonce du diagnostic, la mise en œuvre de l'aide, etc.). Elle suit donc l'évolution des troubles neurodégénératifs et impose de concevoir les difficultés des aidants selon une approche longitudinale, qui tienne compte de l'évolution de la relation aidant-aidé. C'est une histoire d'aidant et d'aidé, au cours de laquelle le rôle acquis évolue au gré des crises et des pertes et qui renvoie au sens de l'existence. Ainsi, de même qu'une approche du vieillissement centrée sur la cognition a montré ses limites [47], une représentation des aidants trop fonctionnaliste apparaît aussi comme résolument limitée. Il incombe désormais d'intégrer mieux les approches relationnelles aux prises en charges à destination des aidants. Pour améliorer leurs effets modestes [48], il s'agira particulièrement de permettre aux aidants de faire face à leurs désaccords, ou à leurs attentes divergentes et donc de leur permettre de faire preuve de créativité et de souplesse relationnelle [49]. Comme l'ont affirmé Schuster et Pellerin [50], la société se doit aujourd'hui de garantir aux aidants leur liberté, y compris d'endosser ce rôle, sans qu'ils éprouvent de culpabilité lorsqu'il s'agit de les remplacer, mais aussi en pensant ce rôle selon ses évolutions.

Conflit d'intérêt : Aucun. 


\section{Références}

[1] Blanpain N, Chardon O. Projections de population à l'horizon 2060. Un tiers de la population âgé de plus de 60 ans. INSEE Prem 2010; 1320. http://www.insee.fr/fr/themes/document.asp?reg_id=0\&ref_id=ip1320

[2] Wolfs CA, Kessels A, Severens JL, et al. Predictive factors for the objective burden of informal care in people with dementia: a systematic review. Alzheimer Dis Assoc Disord 2012;26(3):197-204.

[3] Stensletten K, Bruvik F, Espehaug B, et al. Burden of care, social support, and sense of coherence in elderly caregivers living with individuals with symptoms of dementia. Dementia 2014;15(6):1422-35.

[4] Riley GA, Evans L, Oyebode JR. Relationship continuity and emotional well-being in spouses of people with dementia. Aging Ment Health 2018;22(3):299-305.

[5] Caron R, Caron L. Le travail psychique de l'accompagnement dans la maladie d'Alzheimer. Encephale 2011;37(6):439-47.

[6] Ostrowski M, Mietkiewicz MC. Du conjoint à l'aidant : l'accompagnement dans la maladie d'Alzheimer. Bull Psychol 2013;3:195-207.

[7] Välimäki T, Vehviläinen-Julkunen K, Pietilä A-M, et al. Life orientation in Finnish family caregivers' of persons with Alzheimer's disease: A diary study. Nurs Health Sci 2012;14(4):480-7.

[8] Yang C-T, Liu H-Y, Shyu Y-IL Dyadic relational resources and role strain in family caregivers of persons living with dementia at home: A cross-sectional survey. Int J Nurs Stud 2014;51(4):593-602. 
[9] Quinn C, Clare L, Woods B. The impact of the quality of relationship on the experiences and wellbeing of caregivers of people with dementia: A systematic review. Aging Ment Health 2009;13(2):143-54.

[10] Ablitt A, Jones GV, Muers J. Living with dementia: A systematic review of the influence of relationship factors. Aging Ment Health 2009;13(4):497-511.

[11] Van Bruggen S, Gussekloo J, Bode C, et al. Problems experienced by informal caregivers with older care recipients with and without cognitive impairment. Home Health Care Serv Q 2016;35(1):11-24.

[12] Meyer J, Mc Cullough J, Berggren I. A phenomenological study of living with a partner affected with dementia. Br J Community Nurs 2016;21(1):24-30.

[13] Hsieh S, Irish M, Daveson N, et al. When one loses empathy: its effect on carers of patients with dementia. J Geriatr Psychiatry Neurol 2013;26(3):174-184.

[14] Sutter M, Perrin PB, Chang YP, et al. Linking family dynamics and the mental health of Colombian dementia caregivers. Am J Alzheimers Dis Other Demen 2014;29(1):67-75.

[15] Shim B, Barroso J, Davis LL. A comparative qualitative analysis of stories of spousal caregivers of people with dementia: Negative, ambivalent, and positive experiences. Int $\mathbf{J}$ Nurs Stud 2012;49(2): 220-9.

[16] Jütten LH, Mark RE, Sitskoorn MM. Empathy in informal dementia caregivers and its relationship with depression, anxiety, and burden. Int J Clin Health Psychol 2019;19(1):12-21.

[17] Keady JD. The dynamics of dementia: A modified grounded theory study. University of Wales, Bangor, 1999. 
[18] Pauline B, Boss P. Ambiguous loss: Learning to live with unresolved grief. London: Harvard University Press, 2009.

[19] Hellström I, Nolan M, Lundh U. Sustaining "couplehood": Spouses' strategies for living positively with dementia. Dementia 2007;6(3):383-409.

[20] Mc Cormack L, Tillock K, Walmsley BD. Holding on while letting go: Trauma and growth on the pathway of dementia care in families. Aging Ment Health 2016;21(6):658-67.

[21] Høgsnes L, Melin-Johansson C, Norbergh KG, et al. The existential life situations of spouses of persons with dementia before and after relocating to a nursing home. Aging Ment Health 2014;18(2):152-60.

[22] Pierron-Robinet G, Bonnet M, Mariage A, et al. Les incidences du sentiment de culpabilité sur la demande d'aide de l'aidant familial. Ann Med Psychol 2018;176(2):170-6.

[23] Mausbach BT, Chattillion EA, Ho J, et al. Why does placement of persons with Alzheimer's disease into long-term care improve caregivers' well-being? Examination of psychological mediators. Psychol Aging 2014 29(4):776-86.

[24] Graneheim UH, Johansson A, Lindgren B-M. Family caregivers' experiences of relinquishing the care of a person with dementia to a nursing home: Insights from a metaethnographic study. Scand J Caring Sci 2014;28(2):215-24.

[25] Schumacher KL. Family caregiver role acquisition: role-making through situated interaction. Sch Inq Nurs Pract 1995;9(3):211-26.

[26] Gaugler JE, Zarit SH, Pearlin LI. The onset of dementia caregiving and its longitudinal implications. Psychol Aging 2003;18(2):171-80. 
[27] McDonnell E, Ryan AA. The experience of sons caring for a parent with dementia. Dementia 2013;13(6):788-802.

[28] Lewis RD. The impact of the marital relationship on the experience of caring for an elderly spouse with dementia. Ageing Soc 1998;18(2):209-31.

[29] Bjørge H, Sæteren B, Ulstein ID. Experience of companionship among family caregivers of persons with dementia: A qualitative study. Dementia 2016;18(1):228-44.

[30] Perry J, O'Connor DL. Preserving personhood: (Re) membering the spouse with dementia. Fam Relations 2002;51(1):55-62.

[31] O'Connor DL. Caring for a memory-impaired spouse: A gender-sensitive perspective. J Women Aging 1995;7(3):25-42.

[32] O'Connor DL. Living with a memory-impaired spouse: (re) cognizing the experience. Can J Aging 1999;18(02):211-35.

[33] O'Shaughnessy M, Lee K, Lintern T. Changes in the couple relationship in dementia care: Spouse carers' experiences. Dementia 2010;9(2):237-58.

[34] Barber CE, Pasley BK. Family care of Alzheimer's patients: The role of gender and generational relationship on caregiver outcomes. J Appl Gerontol 1995;14(2):172-92.

[35] Moen P, Robison J, Dempster-McClain D. Caregiving and women's well-being: A life course approach. J Health Soc Behav 1995;36(3):259-73.

[36] Guédeney N. Apport de la théorie de l'attachement à la compréhension de la relation aidant/aidé chez la personne très âgée. Neurol Psychiatr Geriatr 2019;19(109):3-10.

[37] Kramer BJ. Expanding the conceptualization of caregiver coping: The importance of relationship-focused coping strategies. Fam Relat 1993;42(4):383-91. 
[38] Papastavrou E, Tsangari H, Karayiannis G, et al. Caring and coping: The dementia caregivers. Aging Ment Health 2011;15(6):702-11.

[39] Bergman M, Graff C, Eriksdotter M, et al. The meaning of living close to a person with Alzheimer disease. Med Health Care Philos 2016;19(3):341-9.

[40] Leite M, Almeida V, Gonçalves MP. Predictive study of psychological variables impact in family caregivers of patients with Alzheimer. Int J Appl Math Stat 2015;5(6A):614.

[41] Crispi EL, Schiaffino K, Berman WH. The contribution of attachment to burden in adult children of institutionalized parents with dementia. Gerontologist 1997; 37(1):52-60.

[42] Nelis SM, Clare L, Whitaker CJ. Attachment in people with dementia and their caregivers: A systematic review. Dementia 2014;13(6):747-67.

[43] Chen CK, Uzdawinis D, Schölmerich A, et al. Effects of attachment quality on caregiving of a parent with dementia. Am J Geriatr Psychiatry 2014; 22(6) : 623-31.

[44] Monin JK, Schulz R, Kershaw TS. Caregiving spouses' attachment orientations and the physical and psychological health of individuals with Alzheimer's disease. Aging Ment Health 2013;17(4):508-16.

[45] Chen CK, Bailey RW. Episodic memories of relationship quality, procedural knowledge of attachment scripts, and the experience of daughters caring for a parent with dementia. Dementia 2016;17(1):61-77.

[46] Wang C-L, Shyu Y-IL, Wang J-Y, Lu C-H. Progressive compensatory symbiosis: spouse caregiver experiences of caring for persons with dementia in Taiwan. Aging Ment Health 2017;21(3):241-52. 
[47] Whitehouse PJ, George D. Le mythe de la maladie d'Alzheimer. Marseille: Solal, 2009.

[48] Wennberg A, Dye C, Streetman-Loy B, et al. Alzheimer's patient familial caregivers: A review of burden and interventions. Health Soc Work 2015;40(4),e162-e169.

[49] Lethiais L, Leborgne K. La thérapie familiale comme approche contextuelle en psychogériatrie. Neurol Psychiatr Geriatr 2008;8(46):27-9.

[50] Schuster JP, Pellerin J. La relation aidant-aidé: la soutenir, toujours la soutenir. Neurol Psychiatr Geriatr 2019;19(109);11-5. 